\title{
Belief functions and uncertainty management in networks and telecommunication
}

\author{
Abdelhamid Mellouk • Latifa Oukhellou • Lei Shu • \\ Glenn Shafer
}

Published online: 18 February 2014

(C) Institut Mines-Télécom and Springer-Verlag France 2014

In the last few years, Dempster-Shafer theory also known as Theory of Belief Functions (TBF) or Evidence theory has received growing attention in many fields of applications such as finance, technology, biomedicine, etc. This theory may be seen as a generalization framework of different instances such as probability, fuzzy sets, and possibility theories. Using Dempster-Shafer belief functions to express available information allows considering two kinds of uncertainty: aleatory uncertainty due to the variability of the variable of interest in the population and epistemic uncertainty due to a lack of knowledge on the state of the variable.

Different sources of uncertainty and imprecision may arise in network and telecommunication domains. Such imperfection may be due to imprecision of many aspects regarding the

\author{
A. Mellouk $(\bowtie)$ \\ Department of Network \& Telecommunication and LiSSi \\ Laboratory-TincNET Team, University of Paris Est Créteil Val de \\ Marne (UPEC), 122, rue Paul Armangot, 94400 Vitry sur \\ Seine, France \\ e-mail:mellouk@u-pec.fr \\ L. Oukhellou \\ COSYS - GRETTIA, IFSTTAR-French Institute of Science and \\ Technology for Transport, Development and Networks, \\ 14-20 Boulevard Newton, Cité Descartes, Champs sur Marne, \\ 77447 Marne la Vallée Cedex 2, France \\ e-mail: latifa.oukhellou@ifsttar.fr \\ L. Shu \\ Guangdong Provincial Key Lab. of Petrochemical Equipment Fault \\ Diagnosis, Guangdong University of Petrochemical Technology, \\ No. 139, Guandu Erlu, Maonan, Maoming, Guangdong, China \\ 525000 \\ e-mail: lei.shu@lab.gdupt.edu.cn

\section{G. Shafer} \\ Rutgers Business School-Newark and New Brunswick, \\ 1 Washington Park, Newark, NJ 07102, USA \\ e-mail: gshafer@business.rutgers.edu
}

environment: signal, data link, network, etc. For example, it may be due to communication links that might be unreliable, either due to operational tolerance levels or environmental factors. As detailed in the survey paper proposed par Mustapha Reda Senouci, Abdelhamid Mellouk, Mohamed Abdelkrim Senouci, and Latifa Oukhellou in this special issue, the Theory of Belief Functions has proved to be particularly useful to represent and reason with partial information in a wide range of applications, including signal processing, coding, supervision, localization, resource provisioning, etc. In such case, the belief function theory provides a flexible framework for handling and mining imprecision and uncertainty as well as combining different disparate evidence about uncertain events. Indeed, this theory allows modeling different concepts such as imprecision, ambiguity, and ignorance. Also, a variety of combination operators is available in the fusion process.

This special issue of Annals of Telecommunications is intended to provide the recent advances on the use of the Theory of Belief Functions and machine learning approaches in telecommunication and network technologies. It focused on how belief functions and machine learning have affected different aspects (protocols, algorithms, paradigm, energy, signal coding, etc.) for a large family of applications (healthcare, medical, underwater, vehicular, robotic, etc.) using network technologies (sensor networks, MANET, VANET, etc.).

This special issue starts with papers dedicated to the general problem of uncertainty management in telecommunication and networks. They cover several topics as varied as traffic identification, routing, and synchronization/decoding. Frederic Launay and Patrick Coirault address the problem of synchronization and decoding of quasi-chaotic signals. They propose a new approach based on belief propagation algorithm. A second-order Markov model is used to synchronize a quasi-chaotic sequence. Two algorithms are considered, namely, the Viterbi algorithm and the backward-forward algorithm. Based on a combined use of multifractal analysis of 
wavelet energy spectrum and neural network classifiers, Hongtao Shi, Gang Liang, and Hai Wang propose a novel traffic identification approach which does not require any payload information. Different application traffic identifications are achieved by performing classification over the wavelet energy spectrum coefficients that were inferred from the original traffic.

The problem of human activity recognition in the framework of belief functions is then investigated by Faouzi Sebbak, Farid Benhammadi, Abdelghani Chibani, Yacine Amirat, and Aicha Mokhtari. In this paper, the authors present a framework based on Dempster-Shafer Theory to tackle the problem of human activity recognition in smart home environments. The proposed methodology allows converting and combining the raw data captured using a wireless sensor network into highlevel activity knowledge. It allows also optimizing decisionmaking in the presence of conflicting activities.

Considering vehicular ad hoc networks, Mira Bou Farah, David Mercier, Eric Lefevre, and Francois Delmotte propose two models based on belief functions for exchanging and managing uncertain events on the road. The main purpose of this application is to provide the most reliable information for the driver from multiple uncertain messages received about events on the road. The proposed approach allows to manage imperfect information about events on the road in vehicular ad hoc networks. The experiments are carried out through various simulations. An implementation with Android smartphones using a Bluetooth technology to exchange the messages is also introduced in the paper.

The remaining papers address the problem of routing. A novel energy-aware clustering algorithm for the Optimized Link State Routing (OLSR) is proposed by Ahmed Loutfi, Mohammed Elkoutbi, Jalel Ben Othman, and Abdellatif Kobbane. The authors present a clustering approach that elects a reduced and reasonable number of cluster heads that have a high residual energy. Their solution can prolong the lifetime of the entire network and enhance the routing process. The paper proposed by Nadeem Javaid, Ayesha Bibi, Akmal Javaid, Zahoor Ali Khan, Kamran Latif, and Mohammad Ishfaq investigates the quality routing metrics in wireless multi-hop networks. The authors consider three existing quality link metrics, namely, Expected Transmission Count (ETX), Minimum Delay (MD), and Minimum Loss (ML), and they propose a new one, called Inverse Expected Transmission Count (InvETX), with the aim to enhance the conventional Optimized Link State Routing (OLSR) protocol. Simulations are carried out to compare all these quality metric results for conventional and enhanced OLSR.

The last paper, proposed by Abdullah-Al-Wadud, Md. Abdul Hamid, and Ilyoung Chong, investigates the particular problem of fault-tolerant structural health monitoring protocol using wireless sensor networks in the framework of DempsterShafer theory. By discounting the unreliable observer's measurements, the authors show significant improvements in terms of detection accuracy compared to bayesian approaches.

We thank all anonymous reviewers for their hard work, time, and support that greatly helped us select the best papers for this special issue. We also thank all authors who submitted their papers for the consideration for this issue. We express our gratitude to the staff of Annals of Telecommunication for their support and kind encouragements throughout the preparation of this work. Finally, we hope you will enjoy reading this selection of papers as we did.

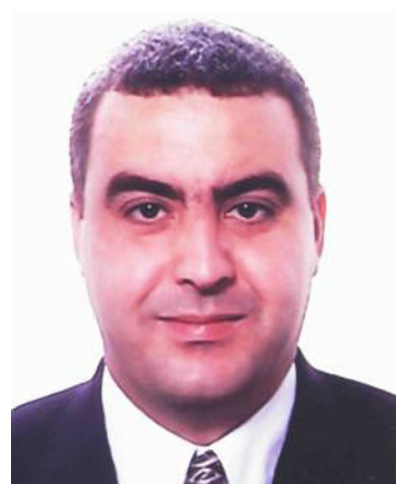

Abdelhamid Mellouk He graduated in computer network engineering from the Computer Science High Engineering School, University Oran-EsSenia, Algeria, and the University of Paris Sud XI Orsay. He received the $\mathrm{PhD}$ degree in informatics from the same university, and the Doctorate of Sciences (Habilitation à Diriger les Recherches) diploma from University of Paris-Est (UPEC). He is a full professor at the Networks \& Telecommunications (N\&T) Department and LiSSi Laboratory, UPEC, France. He is the founder of the Network Control Research activity in UPEC with extensive international academic and industrial collaborations. His general area of research is in adaptive real-time control for high-speed new generation dynamic wired/wireless networking in order to maintain acceptable quality of service/experience for added value services. He is an active member of the IEEE Communications Society and held several offices including leadership positions in IEEE Communications Society Technical Committees (The Technical Committee on Communications Software, The Technical Committee on Switching and Routing). He is a senior member of IEEE.

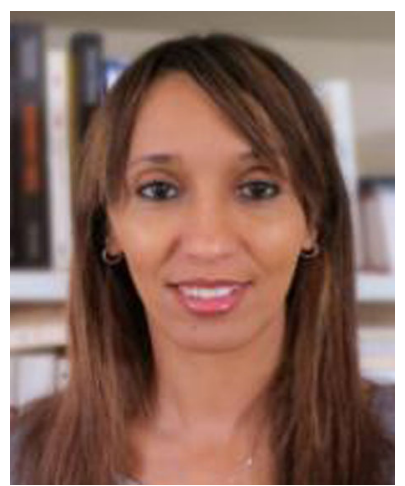

Latifa Oukhellou She received a Ph.D. from Paris-Sud University (Automatic and Signal Processing) in 1997 and $\mathrm{Ha}$ bilitation à diriger des Recherches from Paris-Est University in 2010. She is currently a senior researcher at the French institute of science and technology for transport, development and networks (IFSTTAR) and has been an assistant professor at the University of Paris-Est 
Créteil (UPEC). She is a head of the Diagnosis and maintenance group at the Grettia laboratory (Engineering of Surface Transport Networks and Advanced Computing) of IFSTTAR. Her research interests concern pattern recognition, machine learning, and information fusion. She has published more than 60 papers in scientific journals and conference proceedings, and she is involved in many research projects.

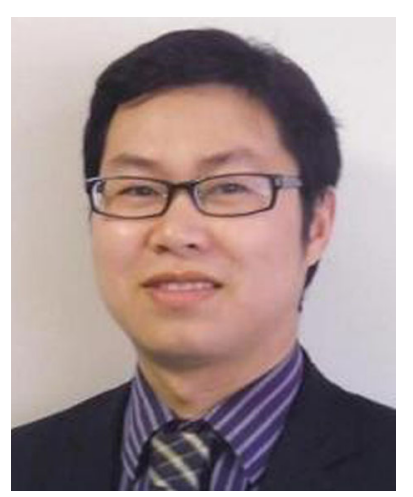

Lei Shu $\mathrm{He}$ received a Ph.D. degree from the Digital Enterprise Research Institute, National University of Ireland, Galway, Ireland in 2010. Since October 2012, he joined Guangdong University of Petrochemical Technology, China as a full professor. Since 2013, he started to serve in Dalian University of Technology as a Ph.D. supervisor. Meanwhile, he is also working as the vice-director of the Guangdong Provincial Key Laboratory of Petrochemical Equipment Fault Diagnosis, China. $\mathrm{He}$ is the founder of the Industrial Security and Wireless Sensor Networks Lab. His research interests include the following: wireless sensor networks, multimedia communication, middleware, and security. He has published over 170 papers in related conferences, journals, and books in the area of sensor networks. He had been awarded the Globecom 2010 and ICC 2013 Best Paper Award. He has been serving as Editor in Chief and editors of many journals and has served as Co-Chair for more than 50 various international conferences/workshops.

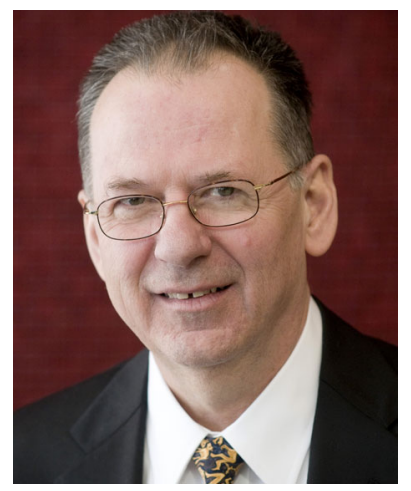

Glenn Shafer He has been a university educator for 40 years. He completed his Ph.D. in mathematical statistics at Princeton University in 1973. As a scholar, he is best known for his work in the 1970 s and 1980 s on the Dempster-Shafer theory, an alternative theory of probability that has been applied widely in engineering and artificial intelligence. The Belief Functions and Applications Society, which is devoted to the advancement of this theory, has been holding international conferences since 2010 .

Shafer is also known for his initiation, with Vladimir Vovk, of the gametheoretic framework for probability. They are now preparing a second edition of their book on the topic, Probability and Finance: Its' Only a Game! The third international workshop on game-theoretic probability was held in Tokyo in 2012. In 2009, Shafer was recognized for his work on these topics with an honorary doctorate in economics by the University of Economics, Prague. Shafer has been the Dean of the Rutgers Business School-Newark and New Brunswick since January 2011. In his nearly 3 years as the Dean, he has overseen a large expansion of the school's undergraduate programs on its two campuses and launched new programs at the master's level in marketing research, supply chain management, financial analysis, and business analytics. The school has strengthened its mentoring programs for undergraduates, it career placement services at both the undergraduate and graduate levels, and its programs for serving all of New Jersey's students, including women and underrepresented minorities. Shafer has also strengthened the school's connections with the business community and raised its stature by expanding support for faculty research. Shafer spent his childhood on a farm near Caney, KS, earned an undergraduate degree in mathematics at Princeton, and served in the Peace Corps in Afghanistan before launching his academic career. Before joining the Rutgers Business School in 1992, he taught at Princeton and the University of Kansas. He has been a Guggenheim Fellow, a Fulbright Fellow, and a visiting professor in Paris and Berlin. In 2004, he received Rutgers' most prestigious faculty award, the Gorenstein Award for Research and Service, and he is one of two dozen Rutgers faculty members recognized as Board of Governors Professors. Since 2002, Glenn and his wife Nell Painter, artist and distinguished professor emerita of history at Princeton University, have lived in Newark, New Jersey's largest and most vibrant city. Professor Painter's most recent book is The History of White People. 